\title{
A Case Report on Cetirizine Induced Urticaria
}

\author{
Lucca JM1* ${ }^{*}$, Thomas $\mathrm{D}^{2}$, Jaimy $\mathrm{A}^{3}$ and Srinath ${ }^{4}$ \\ ${ }^{1}$ Assist. Professor, College of clinical Pharmacy, Imam Abdulrahman Bin Faisal \\ University, KSA \\ ${ }^{2}$ PharmD Intern, Department of Pharmacy Practice, JSS College of pharmacy, India \\ 3Intern, Department of Pharmacy Practice, JSS College of pharmacy, India \\ ${ }^{4}$ Department of medicine, JSS Hospital \& Medical College, India
}

\section{Case Report}

Volume 3 Issue 2

Received Date: February 17, 2018

Published Date: March 08, 2018

DOI: $10.23880 /$ cdoaj- 16000143

*Corresponding author: Jisha M Lucca, Assist. Professor, College of clinical Pharmacy, Imam Abdulrahman Bin Faisal University, Dammam, KSA, Tel: +966 0558181537; E-mail: jmlucca@iau.edu.sa

\section{Abstract}

Cetirizine, anti-histamine is a widely used OTC medication for the treatment of hypersensitivity reactions like urticaria. Cetirizine induced hypersensitivity reactions are rarely reported. Here is a case of cetirizine induced multiple episodes of urticaria in a patient with fever with headache.

Keywords: Anti-histamine; Cetirizine; Urticaria

\section{Introduction}

Cetirizine is widely used as an OTC antihistamine for all type of allergy. Its antihistaminic activity is by the selective inhibition of the peripheral $\mathrm{H}_{1}$ receptors [1]. Somnolence, fatigue, headache, dry mouth and dizziness are the frequently reported adverse effects of cetirizine [2]. Cetirizine induced urticaria are very rare. An antihistamine indicated for urticaria, causing multiple episodes of urticaria make this case more interesting.

\section{Case History}

A 53year old married female patient from a rural background visited her neighbor friend, who is a nurse, for fever and headache. She was given with tablet cetirizine $10 \mathrm{mg}$ one at night. After two hours, she developed generalized itching and consulted a local physician. An impression of allergic reaction was made and symptomatically treated with tablet chlorpheniramine maleate ( $4 \mathrm{mg}$, thrice daily) and tablet methyl prednisolone (40mg per day). Her symptoms were worsening after taking this medication and she revisited the same physician. Considering her conditions, injection prednisolone 1gm IM was added to the existing therapy. As there was no improvement, she was taken to a tertiary care hospital by the family members. All physical and laboratory parameters were analyzed and a provisional diagnosis of 'Drug induced Urticaria' was made. All her medications were withheld. Later patient was referred to the clinical pharmacist, where a detailed medication history interview was taken. Patient revealed that she was a known case of hypertension and diabetes since seven years and on tablet telmisartan + chlorthalidone $(40+12.5 \mathrm{mg}$, once daily), tablet glimipiride + metformin (1g+500mg,once daily) for the last three years. She gave a past history of urticaria after taking cetirizine for the same indication. 


\section{Discussion}

Urticaria describes a cluster of conditions in which there are wheals (or weal's) in the skin i.e., itchy white or red lumps. The most common causes include certain foods, medications, infections, insect bites and internal diseases. Medications reported to be linked with urticaria comprises Antibiotics, NSAID's, Opiates, Antihypertensive's, Antidepressants, etc [3]. Incidence of drug induced urticaria or angioedema varies from $0.16 \%$ to $9 \%$ in various department including dermatology and medicine [4]. The first line agent for drug induced urticaria is antihistamine and cetirizine is the commonly used cheap drug among them. Cetirizine, a human metabolite of hydroxyzine reduces the histamine release by antagonizing the active and selective $\mathrm{H}_{1}$ receptor. The mechanism of cetirizine in urticaria involves inhibition of PAF-induced influx of eosinophils [5].

The latent period of drug induced urticarial symptoms usually occur within $24 \mathrm{hrs}$ after the administration of the drug, which is comparable to our patient's presentation [4]. There are very limited reports on cetirizine induced urticaria and the mechanism by which urticaria occurs is unknown. It is evident that a good prognosis of urticaria can be achieved after treatment with chlorpheniramine maleate [5]. But in this case there was worsening of symptoms with the use of CPM. So she was prescribed with injection methyl prednisolone $1 \mathrm{gm}$ IM. The rational use of steroids is for the stabilization of mast cell membranes and thereby further inhibition of histamine release, as well as reduction in the inflammatory effect of histamine and other mediators [2].

In this case, cetirizine induced urticaria is confirmed, as the patient had a past history of cetirizine induced urticaria several times. At the same time, patient had been taking anti-hypertensive and anti- diabetic medication since three years and gives a safe history, thus these drugs can be excluded. Based on literature search, patient medication history and time temporal relationship, Cetirizine is suspected to be the casual agent for this adverse reaction.

\section{Conclusion}

Even though cetirizine has a well tolerated safety profile, there is exceptionally rare chance of it causing urticarial symptoms. Cetirizine can mimic the underlying disease for which it has been indicated. Therefore, the likelihood in the differential diagnosis of a patient not responding to treatment or further worsening of symptoms has to be considered. Hence this case report is alarming to all the health care professionals who use medicines for an off label indication.

\section{References}

1. Marcia L Buck (2010) Cetirizine and Levocetirizine Use in Children. Pediatr Pharm 16(6).

2. Henry K Wong (2016) Acute Urticaria Treatment \& Management.

3. Natasha Casie Chetty, Clive EH Grattan (2010) What to look for with drug induced urticaria.

4. Shipley D, Ormerod AD (2001) Drug-induced urticaria. Recognition and treatment. Am J Clin Dermatol 2(3): 151-158.

5. Bhargava $\mathrm{P}$, Bhargava S, Kuldeep CM, Mathur NK (1997) Cetirizine induced urticaria. Indian J Dermatol Venereol Leprol 63(1): 71-72. 